\title{
Urban Expressway Travel Time Prediction Method Based on Fuzzy Adaptive Kalman Filter
}

\author{
Yanguo Huang ${ }^{1,2}$, Lunhui Xu ${ }^{1}$, Qiang Luo ${ }^{1}$ and Xianyan Kuang ${ }^{2}$ \\ ${ }^{1}$ School of Civil Engineering and Transportation, South China University of Technology, 510640 Guangzhou, Guangdong, China \\ ${ }^{2}$ Electrical Engineering and Automation Institute, Jiangxi University of Science \& Technology, 341000 Ganzhou, Jiangxi, China
}

Received: 3 Oct. 2012, Revised: 26 Dec. 2012, Accepted: 28 Dec. 2012

Published online: 1 Jun 2013

\begin{abstract}
According to the poor adaptive ability of traditional filter algorithm in the estimation for traffic state and travel time with Kalman filter, an improved fuzzy adaptive Kalman filtering method was proposed. The new interest of observation noise was defined, and the fuzzy logic was used to adjust the importance weights of system noise and observation noise through on-line monitoring the interest changes, which changed the trust and utilization degree of the model for the observation information, and this made the filter eventually tend to be stable. To guarantee the real-time performance of system, a direct input - output fuzzy membership function matching method was put forward to take the place of fuzzy reasoning. The method was tested on the urban expressway in Guangzhou by using real-time detection data, and the result show that the traffic state estimation model had better tracking ability than conventional Kalman filter, and results of travel time prediction show that there was a slight difference between the prediction value and that of actual observation in free traffic flow state, and the relative error was under $15 \%$ in traffic congested state. The precision and applicability of this method were acceptable, and it can be used to provide a basis for travel time of urban expressway in traffic control and guidance system.
\end{abstract}

Keywords: Urban expressway, travel time prediction, adaptive Kalman filter, fuzzy logic, traffic volume

\section{Introduction}

Travel time is one of the comprehensive index which can be used evaluate the road traffic state, and reflect the whole road smooth degree and transportation efficiency. Dynamic route travel time estimation and forecast in different traffic condition can provide the travel time information for travelers from source to destination. The travelers can make their travel plan, choose different traffic mode, travel path and starting time, and they can avoid crowded sections, save travel time and improve the trip efficiency.

Some methods have been used to travel time prediction, according to prediction principle, they could be divided to three class. The first was mathematical statistics and analysis method based on the traditional mathematical and physical model, such as time series model [1,2], parameters regression model [3] and exponential smoothing model [4] and so on. The second method used artificial intelligence optimization method, i.e. genetic algorithm, state space reconstruction model, neural network, and support vector machine (SVM) $[5,6]$. The third was to establish mathematical model based on the traffic flow theory, then analyzed and verified the model through traffic simulation [7]. The difference between the latter with the former two methods was whether can establish system model. The method based on machine learning has high precision prediction, and it needn't build real model which reflect physical characteristics of the system. The neural network model has a good learning ability, and can track fitting nonlinear and non-stationary time series data, but it need a large amount of historical data. SVM is a new tool to solve machine learning problem by means of optimization methods, and it has the complete statistical learning theory basis and excellent learning ability $[8,9]$. In recent years it has been used in the bus travel time prediction [10], but it is difficult to online predict road travel time. The Kalman filter is a kind of recursion linear minimum variance estimate, which uses dynamics equation (state equation) to describe the dynamic change regulation of estimated parameters [11]. Many scholars used this

*Corresponding author e-mail: jxhuangyg@126.com 
method to forecast the route travel time, and obtained satisfactory results [12]. While in the application of standard Kalman filter, if want to gain ideal filtering effect, the condition must be met. It requires that the system model and measurement model are known, and the statistical characteristics of system noise and measurement noise must be zero mean and white noise. In the practical application, due to the randomness characteristics of traffic system and the error of the detector, noise estimation changes with time. If these prior information are insufficient or inaccurate, it would make estimation precision of filter reduce, and even causes filter divergence. Therefore, as for randomness and complex system, in order to achieve better prediction effect, noise intensity need to be timely adjusted.

In this paper, the Kalman filter was used to predict road travel time. Firstly, the travel time characteristic of urban expressway was analyzed, and the Kalman filter prediction model for travel time was established. Then after analysis of the effect of noise to the stability of Kalman filter, an adaptive Kalman filtering algorithm based on fuzzy logic was proposed, which used fuzzy logic controller to online adjust the Kalman filter parameters, and prevented the filter divergence. Finally, a typical expressway in Guangzhou was as an example to verify the validity of this method.

\section{Urban Expressway Travel Time Analysis}

Urban expressway is a continuous passing special road for car. All entrances and exits of it are controlled, where it interests with the main road is the three-dimensional cross. There is no light control, and the design speed is about $60 \sim 100 \mathrm{~km} / \mathrm{h}$. In a city, there are many intersections between expressway and different grade roads, which make the traffic conversion more frequently, and the distance between the entrances and exits of the expressway is obviously less than highway. At the same time, the adjacent mixed zone is very near, and it makes the traffic organization more complex at the entrances and exits, which are the main factors that affect the capacity and service level of expressway. Therefore, during in the travel time prediction for expressway, not only the current traffic flow of the section, occupancy, history traffic state, traffic volume of upstream relevant sections and traffic accidents should be considered, but also the continuous and fast characteristic of the expressway and smaller space between the entrances and exits. These factors determine that the specific model and method are needed to predict the travel time of urban expressway [13].

One urban expressway is made up of entrances, exits and sections, and the travel time of an expressway is the combined by a large number of basic link travel time. This paper mainly analyzed a link travel time.

Definition: Basic link of a road. A typical link of an expressway contains an entrance and an exit and a section

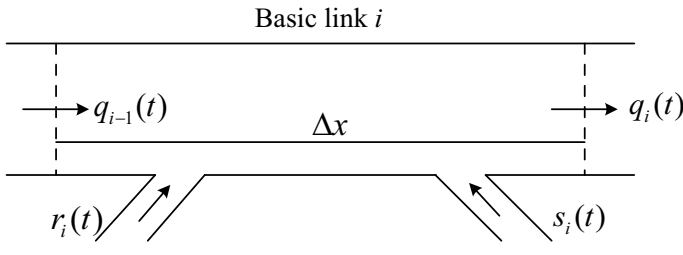

Fig. 1: Basic link of an expressway.

of a road, and the length is more than $1.2 \mathrm{~km}$, as shown in Fig. 1.

In the period of time $(t-1, t)$, the traffic volume that vehicles go through the link $i$ can be estimated as fellows:

$$
q(t)=\alpha\left[q_{i-1}(t)+r_{i}(t)\right]+(1-\alpha)\left[q_{i}(t)+s_{i}(t)\right]
$$

While $q_{i-1}(t)$ is the traffic volume of upstream section, and $q_{i}(t)$ is that of downstream in the period $(t-1, t) . r_{i}(t)$ and $\mathrm{s}_{i}(t)$ is respectively vehicle number which entrance ramp and exit ramp during the time $(t-1, t) . \alpha$ is smoothing factor, and in this paper, the $\alpha$ was taken 0.5 .

According to the traffic flow theory, there are basic relations among the traffic velocity $v(t)$, traffic flow rate $q(t)$ and density $k(t)$.

$$
q(t)=k(t) * v(t)
$$

Assume that the traffic flow in the section is evenly distributed, $q_{i}(t)$ and $v_{i}(t)$ is respectively the traffic volume and speed of the section, then the link travel time can be estimated as fellow:

$$
T i(t)=\frac{L i}{v i(t)}=\frac{L i}{q i(t)} k i(t)
$$

While $L_{i}$ is the length of the link. The link travel time is closely related with the traffic density $k(t)$, and the density can be expressed as the fellows:

$$
k i(t)=k i(t-1)+\frac{1}{N L i}\left\{q_{i-1}(t)+r_{i}(t)-\left[q_{i}(t)+s_{i}(t)\right]\right\}
$$

While $N$ is number of main lane. In the practical application, there are many loop coils at the entrances and exits of expressway, which detect vehicle and collect traffic parameters such as traffic volume and speed. In formula (4), the current density of the link is related with density of the last moment and the current traffic volume. If the traffic state of last moment has been known, the current traffic state could be predicted, and the link travel time could be estimated through the formula (3).

\section{Travel time prediction based on fuzzy Kalman filter}

\subsection{Kalman filter prediction model}

Kalman filter is a filtering algorithm which extracts the estimate value from the observed data in random process 
by linear minimum variance estimation criterion. The basic idea of this method is that the current state can be predicted from the past state according to the state change rule, and use the current observation information to correct the current state. The best feature of this method is able to eliminate random interference noise, and obtain approximation real estimate.

The state equation and observation equation of discrete system are as fellows:

$$
\begin{aligned}
X(k) & =\Phi(k) X(k-1)+B(k) u(k)+G(k) w(k) \\
Z(k) & =H(k) X(k)+v(k)
\end{aligned}
$$

While $X(k)$ is system state variables, and $Z(k)$ is observation vector. $\Phi(k)$ is system state transition matrix, and $H(k)$ is observation matrix. $B(k)$ is input coefficient, and $u(k)$ is input. $w(k)$ is the system noise, and $v(k)$ is measurement noise. They are Gaussian white noise, and independent of each other. The mean value of them is 0 , and covariance is respectively $Q$ and $R$. That is: $E[w(k)]=E[v(k)]=0, \quad E\left[w(k) w^{T}(j)\right]=Q(k) \delta_{k j}$ $\left(\delta_{k j}=1, k=j\right.$; else $\left.\delta_{k j}=0\right)$ and $E\left[v(k) v^{T}(j)\right]=R(k) \delta_{k j}$.

In this paper, Kalman filter was used to predict expressway travel time, and the traffic density of the link $k(t)$ was considered as state variables $X(k)$, and link travel time $T(t)$ was viewed as a measurement variable $Z(k)$. Because the traffic system was a complicated stochastic system, the traffic flow in different traffic conditions exist much randomness. At the same time, there was measurement error in the process of observation. There were the system state noise and observation noise. State noise was assumed to the Gaussian noise, whose mean was $q(t)$, and variance was $Q(t)$. Measurement noise was also assumed to the Gaussian noise, whose mean is $r(t)$, and variance was $R(t)$. The Kalman filter prediction model for a link travel time is as fellows:

$X(k)=\Phi(k) X(k-1)+u(k)$

$Z(k)=H(k) X(k)+v(k)$

While $u(k)=\frac{1}{N L i}\left\{q_{i-1}(k)+r_{i}(k)-\left[q_{i}(k)+s_{i}(k)\right]\right\}$.

The prediction algorithm process is as fellows:

Step 1 status update equation:

$$
\begin{aligned}
X(k \mid k-1) & =\Phi(k \mid k-1) X(k-1 \mid k-1) \\
P(k \mid k-1) & =\Phi(k \mid k-1) P(k-1 \mid k-1) \Phi^{T}(k \mid k-1)+Q
\end{aligned}
$$

Step 2 observation renewal equations:

$$
\begin{aligned}
K(k)= & P(k \mid k-1) H^{T}(k)\left[H(k) P(k \mid k-1) H^{T}(k)\right. \\
& +R(k)]^{-1} \\
\widehat{X}(k \mid k)= & \widehat{X}(k \mid k-1)+K(k)[Z(k)-H(k) \widehat{X}(k \mid k-1)] \\
P(k \mid k)= & {[1-K(k) H(k)] P(k \mid k-1) }
\end{aligned}
$$

If the initial state variable value $X(0)$, initial covariance $P(0)$ and observation value $Z(k)$ are given, the optimal state estimation $X(k)$ of every step is available, and the predicted travel time $Z(k)$ could be obtained.
In the application of standard Kalman filter, the system model and measurement model must be known, and the statistical characteristics of system noise and measurement noise must be white noise, whose means is zero $[14,15]$. When the noise statistical model of system is inaccurate, the precision will be declined obviously, and even lead to Kalman filter divergence. In practical application, due to traffic system randomness, system noise and observation noise estimation change with time. If these priori information is insufficient or inaccurate, they will affect the stability and prediction accuracy of the filter. Therefore, the influence of the changed noise characteristics to filter must be considered. This paper presented an adaptive Kalman filtering algorithm, and this method can online adjust Kalman filter controller by fuzzy logic, which can prevent the filter divergence.

\subsection{Fuzzy Kalman filter design}

The standard Kalman filter demand that the system noise and measurement noise is white noise sequence, and the mean is zero. But in fact, system model can not be exactly accurate, thus it is difficult to determine the prior statistical information of system noise. In dynamic process, the mean of the noise will change, and also the variance $Q(t)$ and $R(t)$. In the Kalman filtering algorithm, the observation noise variance $R$ represents the credibility of the measurement data. With the change of the system state, the $R$ value known in advance is not always credible, and it should change according actual situation. Therefore, it is needed to real-time adjust the Kalman filter noise intensity to achieve the purpose of adaptive control.

Definition: The new interest (residue) was defined as the difference between actual measured value $Z(k)$ and the estimate value $\widehat{Z}(k)$ at $k$ time. It is that

$$
r(k)=Z(k)-H(k) X(k \mid k-1)
$$

The theoretical variance of the new interest is that

$$
\begin{aligned}
\bar{S}(k)= & H(k) P(k \mid k-1) H^{T}(k)+R \\
= & H(k)[\Phi(k \mid k-1) P(k-1 \mid k-1) \Phi(k \mid k-1)+Q] \\
& H^{T}(k)+R
\end{aligned}
$$

If the weight of system noise and observation noise can be real-time adjusted through monitoring new interest (residue) mean and variance changes, the impact of timevarying noise to the filtering stability can be reduced [16].

To choose time window $M$ for new interest sequence statistics smooth, the mean and the variance valuations of new interest at every time can be expressed as:

$$
\begin{aligned}
\bar{r} & =\frac{1}{M} \sum_{j=k-M+1}^{k} r_{j} \\
\overline{S(k)} & =\frac{1}{M} \sum_{j=k-M+1}^{k} r_{j} r_{j}^{T}
\end{aligned}
$$


In an ideal situation, the new interest is Gaussian white noise sequence, and the mean of it is zero. According to the important statistical properties of new interest sequence, it can be used to identify and estimate whether or not the Kalman filter is working in optimal state. If the new interest is not white noise, or the mean of it isn't zero in the operation process, the filter will be in unstable state.

The new interest defined in (14) reflects differences between the predicted value and the observation value. It is additional information for the next step, which reflects the relative change to the prior statistical model of system. New interest is direct information, which is used to on-line adjust prediction model parameter. The noise model can be amended as fellows:

$Q(k)=a(k) Q$
$R(k)=b(k) R$

While $Q$ is initial state noise variance, and is $R$ initial observation noise variance. $a(k)$ and $b(k)$ is respectively the corresponding adjustment coefficient. Then the formula (10) can be modified as fellow:

$$
P(k \mid k-1)=\Phi(k) P(k-1 \mid k-1) \Phi^{T}(k)+a(k) Q
$$

Accordingly, the variance of new interest defined in formula (15) become a function about $a(k)$ and $b(k)$, that is

$$
\begin{aligned}
S(k)= & H(k)\left[\Phi(k) P(k \mid k-1) \Phi^{T}(k)\right. \\
& +a(k) Q] H^{T}(k)+b(k) R
\end{aligned}
$$

The stability of the filter can be online monitored through monitoring the changes of the new interest statistics characteristic. When the stability of the filter is damaged, select proper $a(k)$ and $b(k)$ values to change weight of system noise and observation noise. Thus the reliability and utilization degree of observation information is adaptively adjusted, and the performance of filter can be improved.

The confidence degree of the mean and variance of new interest are defined as fellows:

$m_{1}(k)=\bar{r}(k) \mid Z(k)$

$m_{2}(k)=\operatorname{tr} \bar{S}(k) \mid \operatorname{tr} S(k)$

While the $\boldsymbol{T r}$ is trace operation. When the noise model is accurate, $m_{1}(k)$ value should be near zero, and $m_{2}(k)$ should be near 1 , and the filter state is stable. When the traffic condition changed or traffic accident happened, the actual observation noise increases, and $\bar{r}(k)$ and $\bar{S}(k)$ will also increase. Thus $m_{1}(k)$ will gradually deviate from zero, and $m_{2}(k)$ from 1 . The reliability of previous observation information is reduced, and the filter tends to be unstable. To improve the filter stability, the system noise weight coefficient $a(k)$ and observation noise weight coefficient $b(k)$ must make self-adaption. Under this condition, it is needed to increase the system noise coefficient $a(k)$ and reduce the observation noise coefficient $b(k)$. Conversely, need to reduce $a(k)$ value and increase $b(k)$ value.

Considering the random change of new interest, a fuzzy adaptive adjustment strategy was used to real-time correct noise coefficient by comparing the mean and variance of actual residual with that of theoretical. This paper used fuzzy control logic to adjust parameters. The parameter $m_{1}(k)$ and $m_{2}(k)$ were the input of fuzzy controller, and $a(k)$ and $b(k)$ were output. When $m_{1}(k)$ was around zero, and $m_{2}(k)$ was near 1 , the stability of filter was high. When $m_{1}(k)$ deviated from 0 , and $m_{2}(k)$ from 1 , the stability reduced. In order to real-time predict the link travel time and ensure the accuracy of the results, the fuzzy reasoning method is unfavorable for fuzzy Kalman filter. Therefore, according to the influence characteristics and scale of the changed new interest to the noise model, this paper put forward a method to directly match fuzzy membership degree for input and output variables, and the output $a(k)$ and $b(k)$ is determined as fellows:

$a(k)=1+\eta\left\{F_{\Pi}\left(m_{1}(k)\right)+F_{S}\left(m_{2}(k)\right) / 2\right\}$

$b(k)=\left[F_{\Pi}\left(m_{1}(k)\right)+F_{Z}\left(m_{2}(k)\right)\right] / 2$

While $F_{\Pi}(\cdot)$ is $\Pi$ form fuzzy membership functions, and $F_{S}(\cdot)$ is $S$ form fuzzy membership functions, and $F_{Z}(\cdot)$ is $Z$ form fuzzy membership functions. $\eta$ is a constant, which is determined through testing model in different condition. The parameter selection of fuzzy membership functions should be based on the prior information and specific traffic and road conditions. This paper selected the fuzzy membership functions as shown in Fig. 2, and $\mu$ was fuzzy membership degree.

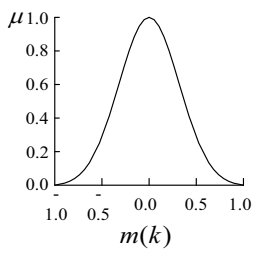

(a) $\Pi$ function

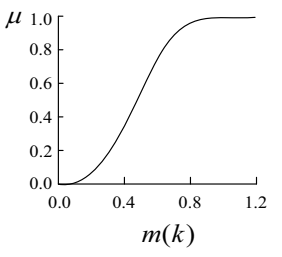

(b) $S$ function

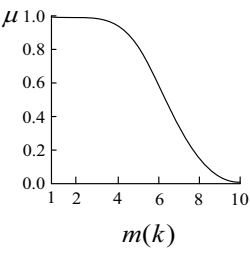

(c) $Z$ function
Fig. 2: Fuzzy membership function of input.

\section{The case analysis}

The Guangyuan expressway in Guangzhou is a standard urban expressway, which used three-dimensional cross at road intersection, and there is no traffic signal control on this way. The length is $47 \mathrm{~km}$, and there are two-way six lanes. The design speed is $60 \mathrm{~km} / \mathrm{h}$ for ground, and 80 $\mathrm{km} / \mathrm{h}$ for viaduct. The distance between adjacent two toll station is about $2 \sim 3.5 \mathrm{~km}$, and there are many traffic detector at the entrance ramp and exit, which is used to detect traffic parameters such as traffic flow, occupy, speed and so on. In this paper, one link of the road was selected as the research object, which has a entrance ramp and a exit ramp. The length of the link is $2.92 \mathrm{~km}$, and 
there is a loop coil detector for every lane. The measured data were used to check traffic state tracking ability of the estimator and prediction effect for expressway travel time.

The traffic data (traffic flow, spend, et al) were got through the loop coil detectors which were installed in the lane. The sampling period was 5 minutes, and 24 hours a day were divided into 288 periods. In order to analyze the relationship between travel time and traffic flow rate and density, three typical times were selected. In the time $0: 0 \sim 6: 00$, the traffic was free running state, and in the time 6:00 9:30, the traffic was under congestion condition. In the 9:00 12:00, the traffic state was relative stability, and traffic flow change was not obvious. In this paper, the traffic data were gotten from charge management station, and the data at 00:00 12:00 on November 22, 2010 were selected to forecast and analyze the results. Two methods were taken to predict the traffic time of the link. The one was standard Kalman filter method, and the other method was to use adaptive Kalman filter based on fuzzy logic. The comparative analysis of the prediction results were made as shown in Fig. 3.

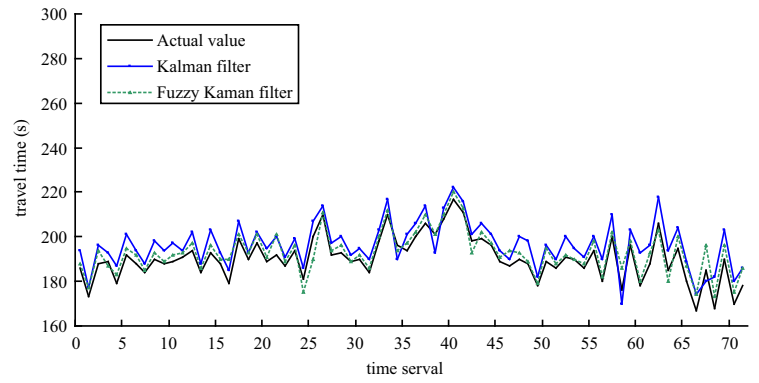

(a) Prediction results in traffic free flow state

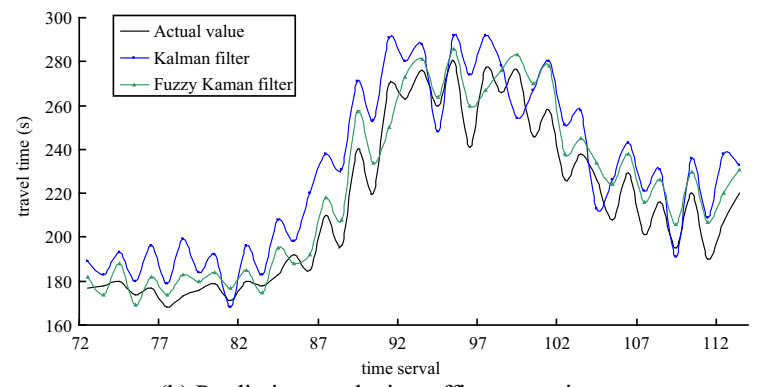

(b) Prediction results in traffic congestion state

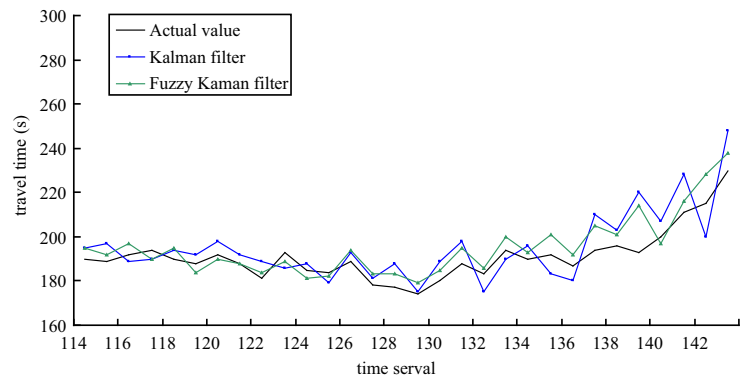

(c) Prediction results in stable traffic flow state

Fig. 3: Results of travel time prediction under different condition.
From Fig. 3(a) and Fig. 3(c), compared with the actual value, the travel time of the two forecast method has the same change trend in the early peak period and peace peak period. But compared with standard Kalman filtering method, the prediction value with adaptive fuzzy control was closer to the actual observation value. As view the results in different periods, with the increase of the traffic flow, the link travel time increased. The main reason of this phenomenon was that the vehicle number on the road in the early peak period was very large, and the traffic state was under congestion, thus the travel speed decreased. On the whole, the prediction results and the measured results had good consistency.

In order to analyze the accuracy and reliability of prediction results under different traffic states, the relative error results analysis was shown in Fig. 4. Compared with the standard Kalman filtering method, the prediction error with fuzzy Kalman filter method was smaller in different traffic condition. It had better stability, and the volatility was obviously less than that of standard Kalman filter method. The error under the free traffic flow state and stable state were small than that of congestion state. The reason was that with the increase of traffic flow, the randomness and uncertainty increased, and system noise and observation noise also increased, which affected the stability of prediction model. If the traffic interference was bigger, such as traffic accidents, there was a lot of volatility of travel time with the standard Kalman filter, while the prediction results based on the fuzzy adaptive Kalman filter was less volatility. The main reason was that adaptive Kalman filtering algorithm could dynamically adjust coefficient of the system noise and observation noise according to the traffic condition.

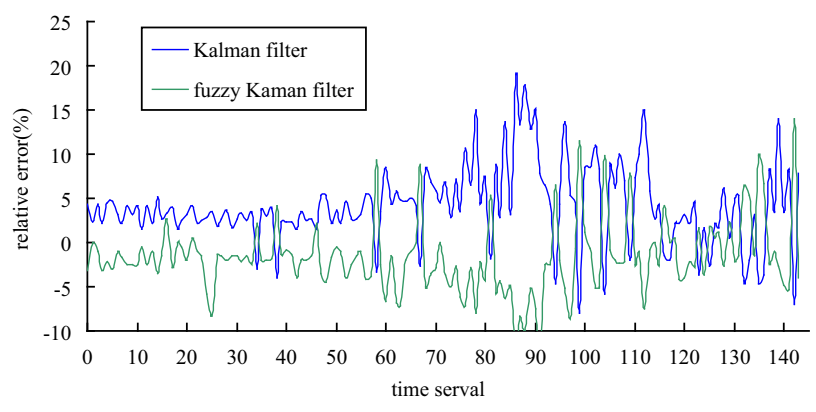

Fig. 4: Comparison of relative error with different prediction methods.

In free traffic flow state, the prediction results were almost completely coincident with the measured data, while the error value was bigger in congestion state. With the change of traffic state, there was hysteresis effect and larger error, but on the whole, it did not affect the reliability of the results, and the error decreased gradually. In few several period, relative differences error of few data were close to $15 \%$, and relative error of most data at other period were below $10 \%$. 


\section{Conclusion}

This paper analyzed the expressway travel time characteristics, established the Kalman travel time prediction model with the traffic density as state variables, and the travel time as observation variable. The complexity and randomness of traffic system make the prior information of system noise and observation noise uncertainty, which affect the accuracy and stability of Kalman filter. To solve this problem, an adaptive Kalman filtering method based on fuzzy logic was put forward. In this method, the variance of system noise and observation noise can be real-time adjusted through monitoring the changes of the new interest mean and variance. The Guangyuan expressway in Guangzhou as a case was analyzed. The results show that compared with conventional Kalman filter, this method has a higher precision. In free traffic flow state and stable traffic flow condition, this method had good prediction accuracy, and the prediction error was below $10 \%$. In the traffic congestion state or under traffic accident, predicted value had certain hysteresis and error fluctuation, but on the whole, it didn't affect the reliability of traffic time prediction. The result of the experiment suggested that this method can better track system state changes, and the filter has good adaptability, which can be used to road travel time real-time dynamic prediction.

\section{Acknowledgements}

This work is supported by the National Natural Science Foundation of China (No.61263024, No.51268017). Thanks for the help.

\section{References}

[1] R. P. Nath, H. J. Lee, N. K. Chowdhury, et al, R. Setchi et al. ( Eds. ) 1, 511-521 (2010)

[2] S. J. Chien and C. M. Kuchipudi, Journal of Transportation Engineering, 608-616 (2003).

[3] J. TU, Y. M. LI and C. L. LIU, Journal of Shanghai Jiaotong University. ( Sci. ) 14, 486-489 (2009).

[4] N. K. Chowdhury, R. P. Nath, H. Lee, et al, J. D. VeláSquez et al. ( Eds. ) 1, 130-138 (2009).

[5] J. Chang, N. K. Chowdhury and H. Lee, Journal of Intelligent \& Fuzzy Systems 21, 5-17 (2010).

[6] T. D. XU, L. J. SUN and Y. HAO, Journal of Tongji University(Nature Science) 36 , 1355-1361 (2008).

[7] H. S. ZHANG, Y. ZHANG and D. C. HU, Journal of Traffic and Transportation Engineering 8, 89-96 (2008).

[8] J. N. Wang, X. M. Chen and S. X. Guo, The $12^{\text {th }}$ International IEEE Conference on Intelligent Transportation Systems, 655-660 (2009).

[9] J. ZHANG and J. SUN, Journal of Transportation Systems Engineering and Information Technology 11, 174-179 (2011).
[10] R. Jeong and L. R. Rilett, Journal of the Transportation Research Board, 195-204 (2005).

[11] Z. X. MA, Z. Y. LIU and M. CHEN, Information and Control 35, 457-461 (2006).

[12] H. H.LIU and Z. S. YANG, Journal of Traffic and Transportation Engineering 8, 88-92 (2008).

[13] J. RICE and E. VAN ZWET, IEEE Transactions on Intelligent Transportation Systems 5, 200-207 (2004).

[14] N. GOU, R. WANG, X. K. GUO and X. L. FENG, Journal of Air Force Engineering University (Natural Science Edition) 8, 36-39 (2007).

[15] J. F. Liu and Z. L. Deng, Applied Mathematics \& Information Sciences 6, 1-7 (2012).

[16] D. LOEBIS, R. SUTTON, J. CHUDLEY and W. Naeem, Control Engineering Practice 12, 1531-1539 (2004).

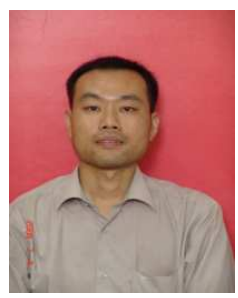

Yanguo Huang, born in 1973, is a $\mathrm{PhD}$ candidate in School of Civil Engineering and Transportation, South China University of Technology, and an associate professor of Jiangxi University of Science \& Technology. The main research field is intelligent transportation system control, traffic system modeling and intelligent optimization algorithm.

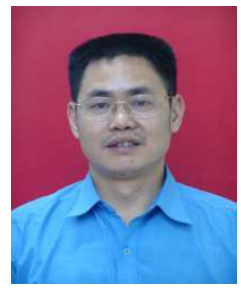

Lunhui Xu, born in 1965, is a professor, doctoral supervisor of School of Civil Engineering and Transportation, South China University of Technology. $\mathrm{He}$ has published more than 80 papers on international journals, national journals, and conferences and 3 books in recent years. His research interests include: intelligent transportation, process control, recognition and artificial intelligence.

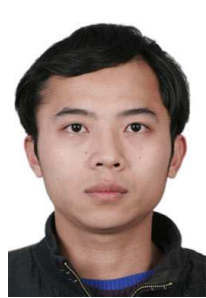

Qiang Luo, born in 1986, is a $\mathrm{PhD}$ candidate in School of Civil Engineering and Transportation, South China University of Technology. His main research interests include intelligent traffic control and traffic safety.

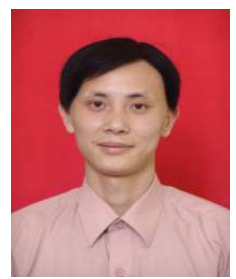

Xianyan Kuang, born in 1976, is a $\mathrm{PhD}$ candidate in School of Civil Engineering and Transportation, South China University of Technology. His main research interests include traffic system modeling and simulation, and intelligent algorithm. 